The abbmitiej monuncript has been nulhered by a cuntractor of the U.S. Guvernment under contrack DE. Acus-etsnzatur. Acrutingly. the U.s. Guverument retalin n Hunexclumive. ruyaliy-free licenare en mublinh or rejoreduce the publiatied form of thin cuntrithtina, ir nllow uthers bu do nu. fut U.S. Guvernment pizrjunca."

CONF-8910225--5

DE90 001334

\title{
ELECTRON HEATING AND STATIC SHEATH ENHANCEMENT IN FRONT OF ENERGIZED RF ANTENNAS*
}

\author{
M.D. CARTER, D.B. BATCHELOR, E.F. JAEGER \\ Oak Ridge National Laboratory \\ Oak Ridge, Tennessee, United States of America
}

\begin{abstract}
DISCLAIMER
This report was prepared as an account of work sponsored by an agency of the United States Government. Neither the United States Government nor any agency thereof, nor any of their employees, makes any warranty, express or implied, or assumes any legal liability or responsibility for the accuracy, completeness, or usefulness of any information, apparatus, product, or process disclosed, or represents that its use would not infringe privately owned rights. Reference herein to any specific commeicial product, process, or service by trade name, trademark, manufacturer, or otherwise does not necessarily constitute or imply its endorsement, recommendation, or favoring by the United States Government or any agency thereof. The views and opinions of authors expressed herein do not necessarily state or reflect those of the United States Government or any agency thereof.
\end{abstract}

* Research sponsored by the Office of Fusion Energy, U.S. Department of Energy, under contract DE-AC05-84OR21400 with Martin Marietta Energy Systems, Inc.

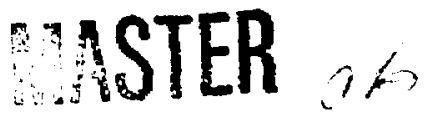


ELECTRON HEATING AND STATIC SHEATH ENHANCEMENT IN FRONT OF ENERGIZED RF ANTENNAS

\section{D. CARTER, D. B. BATCHELOR, E. F. JAEGER}

Oak Ridge National Laboratory, Oak Ridge, Tennessee, United States of America

ABSTRACT. An analytic model has been developed to describe the increased plasma potential and acceleration of ions in the vicinity of an ICRF Faraday shield. This model gives an estimate for the electron distribution function using a heating model with decorrelation caused by reflection of the electrons from the static sheath at the point where a magnetic field line intersects a grounded wall. The loss rate of the electrons from the system can be calculated from this distribution function. The expected static potential is then estimated by equating electron and ion loss rates, assuming either that the ion loss satisfies the Bohm sheath criterion or that ion gyro-orbits strike a nearby wall. The analytic model for the electrons is in good agreement with a Monte Carlo model using realistic values of the RF electric fields. These RF near-field values have been calculated, including the linear near-field plasma response and the Faraday shield. Results include the findings that a linear model for the RF plasma response is valid for typical experimental parameters and that plasma currents must be included in the RF near-field calculation. It is also found that the static potential is sufficiently increased by electron heating so that it could easily cause impurities to be generated from the antenna structure. Results of the expected static potential are given for various ion species.

\section{INTRODUCTION}

The generation of impurities from the Faraday shield and surrounding structures is a commonly observed phenomenon when an ICRF antenna is energized. 
One of the more interesting experiment al observations is the heating of electrons in the region near the antenna $[1,2]$. In this paper, we consider RF near-field effects that occur within a plasma RF skin layer of the Faraday shield in the limit that the RF plasma response is linear. The heating of electrons by the RF near fields is considered by using a quasi-linear electron heating model. The production of high-energy ions can then be understood in terms of an enhanced time-independent (static) sheath near the shield when electrons are strongly heated by evanescent RF electric fields parallel to the static magnetic field, $\vec{B}$, at the locations where the field line intersects a grounded structure. The sheath enhancement results from the fact that the RF excited electron distribution can be far from a collisional Maxwellian. This model demonstrates that the production of impurities in this region can easily result from the high-energy ions that are produced in the low-density plasma near the front face of the Faraday shield.

Meaningful electron excitation occurs only when some phase decorrelation mechanism exists such that electrons can be heated rather than simply oscillating in the RF fields. One decorrelation mechanism that can give rise to electron heating is analogous to Fermi acceleration when observed in a frame that is oscillating with the electron in the parallel RF electric field, $E_{\|}$. In contrast to previous works [3-6] where the amplitude of the sheath oscillates within a few Debye lengths, $\lambda_{d}$, of the wall, we consider an electron that is free to oscillate in the RF electric field except when it reaches a wall where a strong, localized static potential can force the electron to turn before completing an RF cycle. In the oscillating frame, the static sheath that occurs within a few $\lambda_{d}$ of the grounded structure appears to be oscillating in space rather than amplitude. An electron will be reflected by this static sheath if its parallel energy at the time of impact is less than the static sheath potential. Decorrelatirn can oc- 
cur on an electron transit time scale, and the electrons are heated either unt il they overcome the static sheath potential at the end of the field line or until an adiabatic heating limit is reached [7]. The static potential of the magnetic field line increases until the ions in the heated region can maintain quasi-neutrality by escaping via one of two possible mechanisms, depending upon the geometry of the nearby grounded structure. One route for ions to escape is along $\vec{B}$ on a time scale of the order of the connection length divided by the sound speed. The other jon escape route exists if a grounded structure, such as a Faraday shield blade, is nearby, so that the ion strikes it by falling through the perpendicular static potential drop during a gyro-orbit (see Fig. 1). If an ion gyro-orbit strikes a nearby surface, the ion loss rate becomes very fast (of the order of the ion gyroperiod), so that the ions can effectively short out the electron losses along the field line; thus, the static potential within the region dominated by ion gyroorbit losses will roughly obey Laplace's equation. Note that the ion energy upon impact with a grounded surface will be of the order of the floating potential of the heated field line for either of these ion loss mechanisms.

Decorrelation and heating of the electrons could also occur if the mean free path for electron-neutral collisions or Coulomb collusions were shorter than the connection length of the $\vec{B}$ field line; however, we consider only the transit decorrelation mechanism in this paper. This mechanism could heat the edge plasma at locations far removed from the RF antenna if significant amounts of RF power propagate to these regions. The geometry would determine the RF parallel electric field, and the decorrelation mechanism would be determined by the lesser of the connection length between grounded ends of the field line and the collisional mean free path. 


\section{RF ELECTRIC FIELDS PARALLEL TO $\vec{B}$}

The RF antennas used in most experiments are electromagnetically coupled to the plasma. Thus, the source of RF electric near fields is from both changes in the magnetic flux that is squeezed through the slots in the Faraday shield and electrostatic charges that appear on the screen in order to satisfy the boundary conditions. If the antenna is much smaller than a typical free-space wavelength, the electrostatic contributions to the RF fields from the Faraday shield are only comparable to the electromagnetic induction effects. Low-density plasma is likely to be present near the antenna structure, with the density determined by the self-consistent flow of the plasma into limiting surfaces.

We have used the ORION code [8] to solve for the RF fields in the vicinity of a Faraday shield with plasma present. The code includes both RF electrostatic and electromagnetic effects and uses a linear warm plasma dielectric response for specified plasma profiles. Calculations using the code show that, for frequencies near $30 \mathrm{MHz}$ in PLT-type geometries, the electric fields parallel to $\vec{B}$ are small enough to produce a linear plasma response. That is, the electrons in the near-field region are able to freely carry oscillatory RF currents and reduce $E_{\|}$. Results of the ORION code indicate that these plasma currents may not be neglected in the calculation of $E_{\|}$unless the plasma density is very low, in which case the RF electric fields approach the expected vacuum limit.

$E_{\|}$decays away from the Faraday shield and extends into the plasma in the $\boldsymbol{x}$-direction, as shown in Fig. 2. The evanescent scale length of this near-field region is of the order of the plasma skin depth modified by perpendicular profile effects. For the case of PLT-type parameters, these near fields can extend into the plasma for distances of the order of $1 \mathrm{~cm} . E_{\|}$in the gaps at the front face of the Faraday screen reaches values of $10-20 \mathrm{~V} / \mathrm{cm}$ for power levels comparable to 
the experimental values. Significant electron excitation in the near-field region has been observed experimentally with these relatively low values of the parallel electric field : 1$]$

\section{STEADY-STATE STATIC SHEATH MODEL}

The increase of the plasma potential caused by the presence of $E_{\|}$can be estimated by considering a Fermi acceleration heating model for electrons on a field line that intersects a grounded surface. As electron energies increase, adiabatic limits to stochastic heating can be reached when the electron's bounce phase becomes correlated between successive energy kicks. Simple estimates for these limits can be obtained from Ref. [7]. Neglecting any variation in the modulus of $E_{\|}$between the intersection points of the $\vec{B}$ field line with the wall gives an adiabatic energy limit, $W_{\text {lim }}$, of roughiy [7]

$$
W_{l i m}=3 e E_{\|} L
$$

where $E_{\|}$is evaluated at the wall intersection point of the field line and $L$ is the separation distance between the walls. Using $E_{\|}=10 \mathrm{~V} / \mathrm{cm}$ and $L=40$ $\mathrm{cm}$, one finds adiabatic heating limits on the order of keV from Eq. (1). Thus, adiabatic limits are of little concern if $L>10 \mathrm{~cm}$, especially considering the fact that variations in the RF field strength between the walls and collisional eifects would add to the decorrelation of the bounce phase between reflections from the static sheath. Stochastic heating for these RF parameters has also been verified by Monte Carlo modeling.

The electron distribution function can be calculated using a diffusion equation for a stochastic heating model with the diffusion coefficient $D=\nu_{d}\left\langle(\Delta v)^{2}\right\rangle$, where $\nu_{d}=|v| / L$ is the decorrelation rate and $\Delta v=e E_{\|} /(m \omega)$ is the amplitude of the velocity oscillation caused by the RF. Assuming that the predominant potential drop, $\phi$, occurs within a few $\lambda_{d}$ of the wall intersection point of the field 
line and neglecting collisions, the steady-state diffusion of the time-averaged parallel electron velocity in one dimension is described by

$$
\frac{\partial}{\partial v} \frac{|v|}{L}\left(\frac{e E_{\|}}{m \omega}\right)^{2} \frac{\partial f}{\partial v}=0
$$

subject to the boundary conditions that $f=0$ at $v^{2} \geq(2 e \phi / m)$, where $m$ is the electron mass. We note that Eq. (2) is singular at $v=0$; this singularity could be resolved by including the physical effect of Coulomb scattering at very low energies. However, the precise behavior of $f$ at low energy is not important in the calculation of electron losses that occur at energies of $e \phi$, provided that $e \phi \gg T$, where $T$ is a measure of the electron source energy distribution. Thus, we may avoid unnecessary complications in the solution by imagining that the distribution function at low energy is specified by a source of electrons with $T \ll e \phi$. Then, taking the limit that $T \rightarrow 0$, and normalizing to the electron density, $n$, we obtain the resulting electron distribution function,

$$
f=\left(\frac{n}{4}\right) \sqrt{\frac{m}{2 e \phi}} \ln \left(\frac{2 e \phi}{m v^{2}}\right)
$$

and we note that the singularity at $v=0$ is integrable. The electron loss rate through the boundary at $v^{2}=(2 e \phi / m)$ can now be calculated from Eqs (2) and (3) and is given by

$$
\nu_{e}=\frac{1}{L} \sqrt{\frac{m}{2 e \phi}}\left(\frac{e E_{\|}}{m \omega}\right)^{2}
$$

where losses from both ends of the field line are considered. The average energy of the electron distribution is given by

$$
W=\frac{e \phi}{9}
$$


If the ions are constrained by $\vec{B}$ to flow out along the field line, the ion loss rate may be roughly estimated using a modified Bohm sheath criterion [9]. This criterion arrises by considering the fluid equation of motion for a steady-state plasma in one dimension such that the electrostatic force in the presheath region is balanced by the electron pressure gradient force. Assuming an "isothermal" electron response to the presheath potential, the pressure gradient force is given by $(2 W / n) \partial n / \partial z$ and the resulting spatial electron distribution, $n$, in terms of the presheath potential is given by the electron Boltzmann relation with the temperature replaced by an effective temperature of $2 W$. Using this effective temperature and Eq. (5) in the Bohm sheath criterion gives

$$
\nu_{i}=\frac{2}{L} \sqrt{\frac{2 Z e \phi}{9 M}}
$$

where $M$ is the mass and $Z$ is the charge state of the dominant ion species. Setting $\nu_{e}=Z \nu_{i}$ and using Eqs (4) and (6) gives, for a steady-state estimate of $\phi$,

$$
e \phi=e \phi_{a}=\frac{1.5}{Z} \sqrt{\frac{M}{Z m}} W_{\text {osc }}
$$

where $W_{\text {oac }}=\left(e E_{\|} / \omega\right)^{2} /(2 m)$ is the maximum RF oscillation energy of the electron during an RF cycle and the subscript $a$ refers to an acoustic ion response.

If, on the other hand, the ions are able to escape from the system by falling in a direction perpendicular to $\vec{B}$ through the static potential to a nearby grounded surface that is parallel to $\vec{B}$, then the ion loss rate is of the order of the ion gyrofrequency $\Omega_{i}$. This rapid ion loss will allow the potential to drop because the ions can easily flow out across $\vec{B}$ in order to short out the electron flow along $\vec{B}$. Equating $\nu_{e}$ from Eq. (4) and $Z \Omega_{i}$ gives 


$$
e \phi=e \phi_{g}=\frac{H_{o s c}^{-2}}{2 m L^{2} Z^{2} \Omega_{i}^{2}}
$$

where $\phi_{g}$ represents the particular solution to Poisson's equation in the region dominated by jon gyroflow in the direction perpendicular to $\vec{B}$. $\phi_{g}$ is typically very small (of the order $10^{-4} \mathrm{~V}$ ) such that the potential is limited in the $x$-direction to a value $\phi_{m}$ and falls linearly to zero at the grounded wall as determined by Laplace's equation (see Fig. 1).

The value of $\phi_{m}$ can be estimated by assuming an evanescent form for the perpendicular scaling of $E_{\|}(x)$, with scale length $c / \omega_{p e}$. The modulus of $E_{\|}$is then

$$
E_{\|}=E_{\| 0} \exp \left(-x \omega_{p e} / c\right)
$$

where the geometry is illustrated in Fig. $1, \omega_{p e}$ is the electron plasma frequency calculated using some effective edge plasma density to compensate for profile effects, and $c$ is the speed of light in vacuum. An estimate of the peak value of $\phi$ in the $x$-direction is obtained by evaluating $\phi_{m}=\phi_{a}\left(x=x_{g}\right)$ (see Fig. 1), where $x_{g}$ is equal to the gyroradius of an ion with energy given by $Z e \phi_{m} ;$ the ion having fallen through the perpendicular potential drop to strike a nearby grounded surface during a gyro-orbit. Thus, the maximum expected potential in the $x$-direction, $\phi_{m}$, occurring at $x=x_{g}$, is given roughly by the root of the transcendental equation:

$$
\frac{\phi_{m}}{\phi_{0}}=\exp \left(-2 x_{g} \omega_{p e} / c\right)=\exp \left[-\left(2 \rho_{0} \omega_{p e} / c\right) \sqrt{\phi_{m} / \phi_{0}}\right]
$$

with

$$
\rho_{0}=\frac{0.014}{|\vec{B}|} \sqrt{\mu e \phi_{0} / Z}
$$


and

$$
e \phi_{0}=\frac{30}{Z m c^{2}}\left(\frac{e E_{\| 0} c}{\omega}\right)^{2} \sqrt{\frac{\mu}{Z}}
$$

where $\vec{B}$ is the static field in tesla, $\mu$ the ion mass in proton units, and $Z$ the ion charge state; $\phi_{0}$ is in volts. Figure 3 shows the typical solutions to Eq. (10) for various parameters. We note that we have considered only a single species and charge state for the ions in Eq. (10).

Results of this analytic model were compared with a Monte Carlo model using low-energy random initial conditions. For a test case, a deuterium plasma with $E_{\|}=12 \mathrm{~V} / \mathrm{cm}$ at the intersection points of $\vec{B}, \omega / 2 \pi=30 \mathrm{MHz}$, and $L=40 \mathrm{~cm}$ was considered. The value of $\phi_{a}$ from Eq. (7) for these parameters is roughly $300 \mathrm{~V}$, with $W=34 \mathrm{eV}$ given by Eq. (5), and the electron loss rate from Eq. (4) is equal to $3 \times 10^{5} \mathrm{~s}^{-1}$. These RF parameters were used in the Monte Carlo code along with an exponential static potential drop of $300 \mathrm{~V}$ with $\lambda_{d}=0.01 \mathrm{~cm}$ at the field line ends. Initial conditions were sampled trom a $10 \mathrm{eV}$ Maxwellian distribution, and the spatial starting location was chosen at random. The results yielded an electron loss rate of $\nu_{e}=3.2 \times 10^{5} \mathrm{~s}^{-1} \pm 5 \%$ and an average electron energy of roughly $W=35 \mathrm{eV} \pm 5 \mathrm{eV}$. The resulting electron distribution function is shown in Fig. 4 along with the analytic scaling given by Eq. (3). We note that the Monte Carlo result for the distribution function retains some of the Maxwellian initial condition information at low parallel energies. This effect could be included in the analytic estimate by matching $f$ to a Maxwellian with temperature, $T$, at low energy and renormalizing. However, this matching would have only a small effect on the escaping electron flux that is caused by RF heating to relatively high energies if $e \phi$ is greater than a few times $T$. We also nute that the same results (within statistical errors) were obtained for $\nu_{e}$ and $W$ when initial sampling was from a $20 \mathrm{eV}$ Maxwellian distribution using the same RF parameters. 


\section{DISCUSSION}

These estimates can now be used to determine the importance of these processes, assuming that the grounded wall in the perpendicular direction represents the metallic bars of the Faraday shield. Figure 3 shows $\phi_{m}$ and $x_{g}$ irom Eq. (10) as a function of $E_{\| 0}$ for several different singly charged ion species assuming that only one ion species determines the ion loss rate, $\nu_{i}$, in Eq. (6). Note that in a deuterium plasma, deuterium energies on the order of $500 \mathrm{eV}$ would be capable of sputtering a few wall atoms such as nickel. These sputtered nickel atoms could then be ionized and fall through a similar potential drop, impacting the screen with energies in the $500 \mathrm{eV}$ range (if only singly charged) where the self-sputtering yield exceeds unity. The resulting influx of nickel could then influence the sheath potential itself if nickel became the predominant ion species in the region because of its large atomic mass. Assuming a $100 \%$ singly charged nickel plasma, the peak value of the sheath potential drops slightly because of gyro-orbit losses, but the sheath also extends out to a distance of the order of centimeters (the evanescent scale length) away from the Faraday shield. Thus, we see that the experimentally observed sputtering phenomena at the Faraday shield of an energized RF antenna can easily be explained by using realistic values for the pertinent parameters in this model.

The most obvious way to reduce the effects of enhanced static potentials near RF antennas is to reduce $E_{\|}$at the locations where magnetic field lines intersect a grounded structure. These parallel RF fields are typically evanescent, but they can strongly affect the electron distribution function inside the plasma skin depth. Aligning the Faraday shield elements with $\vec{B}$ could help to reduce $E_{\|}$, but special care should be taken to consider any magnetic field lines that intersect any grounded location where large values of $E_{\|}$might be present. We also note that antenna current configurations with dipole current phasings would 
have reduced values of $E_{\|}$near the side walls of the antenna structure because the near-field effects are partially canceled by the dipole phasing. Another way to reduce these fields could be to move the grounded side wall farther away from the current straps, thereby reducing the RF fields where the magnetic field intersects the side walls. In addition, the near fields could be reduced by reducing the Faraday shield impedance.

Another alternative to reducing the ion impact energies might be to somehow enhance the secondary emission of electrons from the grounded locations of the magnetic field lines. The supply of extra electrons onto the heated field line might help to reduce the potential drop, but it might also increase the plasma density and the overall flux of ions to the structure. This option, as well as allowing the DC potential of the antenna structure to float, would require extensive modeling beyond the scope of the model presented here in order to determine the overall effect on impurity production.

If $E_{\text {ll }}$ cannot be sufficiently reduced, a different approach would be to make the antenna structure out of materials with the lowest possible value of $Z_{i} \mu_{i}$, where $Z_{i}$ and $\mu_{i}$ are the charge state and atomic weight of the structural material. The reasoning here is that the lighter the ion, the faster its loss rate along the field line, so that if the material were sputtered, then the loss of ions along the field line would be less likely to result in grazing incidence than perpendicular ion gyro-orbit losses. If bumpers were extended into the plasma in such a way that $E_{\|}$were very small near the bumper ends, most of the sputtered atoms could be redeposited before entering the main plasma, assuming that their mean free path for ionization was less than the distance to the bumper ends. In addition, the impact energy of the impurity ions is $Z_{i} e \phi$, so that heavy atoms that are also easily ionized to $Z_{i}>1$ could impact with very high energies. We note that the average electron energy is actually much less than e $\phi[E q .(5)]$ so that 
ionization to values of $Z_{i}>1$ may not be too severe.

One could also try to reduce the adiabatic electron heating limit by reducing $L\left(L=1 \mathrm{~cm}\right.$ makes $W_{\text {lim }}$ of the order of tens of $\left.\mathrm{eV}\right)$; however, these short values of $L$ would probably have the very undesirable effect of raising $E_{\|}$. On the other hand, the reduction of $L$ would probably reduce the plasma density in the absence of ionization sources and might help if the density became low enough near the screen to reduce the total particle flux.

\section{REFERENCES}

[1] LEHRMAN, I.S., A Study of Coupling and Edge Processes for ICRF Antennas, PhD Thesis, Univ. of Wisconsin, Madison (1988).

[2] CAUGHMAN, J.B.O., The Distribution of Ion Energies Incident on an ICRH Antenna Faraday Shield, PhD Thesis, University of Mlinois, Urbana (1989).

[3] MYRA, J.R., D'IPPOLITO, D.A., GERVER, M.J., Rep. DOE/ER/532633, Lodestar Research Corporation (1989).

[4] PERKINS, F.W., Rep. PPPL-2571, Princeton Plasma Physics Laboratory $(1988)$

[5] EMMERT, G.A., Bull. Am. Phys. Soc. 33 (1988) 1875.

[6] GOEdDE, C.G., LICHTENBERG, A.J., LIEBERMAN, M.A., Rep. UCB/ERL M88/29, University of California at Berkeley (1988).

[7] LiebermaN, M.A., LICHTEnBERG, A.J., Phys. Rev. A 5 (1972) 1852.

[8] JAEGER, E.F., BATCHELOR D.B., CARTER, M.D., WEITZNER H., Global ICRF ware propagation in edge plasma and Faraday shield regions, submitted to Nucl. Fusion. 
[9] BOHM, D., Minimum ionic kinetic energy for a stable sheath, in The Characteristics of Electrical Discharges in Magnetic Fields, McGraw-Hill, New York, (1949) (GUTHRIE, A., WAKERLING, R.K., Eds) 7i-86.

\section{FIGURE CAPTIONS}

FIG. 1. Heating of electrons by RF $E_{\|}$increases the potential of a field line until jons and electrons escape at the same rate. Ion flow may be either along or perpendicular to $\vec{B}$.

FIG. 2. RF electric fields from the ORION code for PLT like geometry. Fields are normalized to $1 \dot{A}$ of antenna current and adjustments for the $k_{\|}$spectrum give roughly $E_{y}=320 \mathrm{~V} / \mathrm{cm}$ for $1 \mathrm{MW}$ of power delivered [1].

FIG. 3. Maximum expected voltages, $\phi_{m}$, and distance from the nearby grounded surface, $x_{g}$, for various values of the ion mass $\mu$ and RF field strengths in the gaps at the front face of the Faraday shield, $E_{\mid 10}$. Other parameters are: $|\vec{B}|=2$ $\mathrm{T}, \omega /(2 \pi)=30 \mathrm{MHz}, c / \omega_{p e}=0.75 \mathrm{~cm}, Z=1$.

FIG. 4. Electron distribution function versus energy from the Monte Carlo code (solid) compares favourably with the analytic result given by Eq. (3) (dashed). 
ORNL-DWG $89 M-2832$ FED
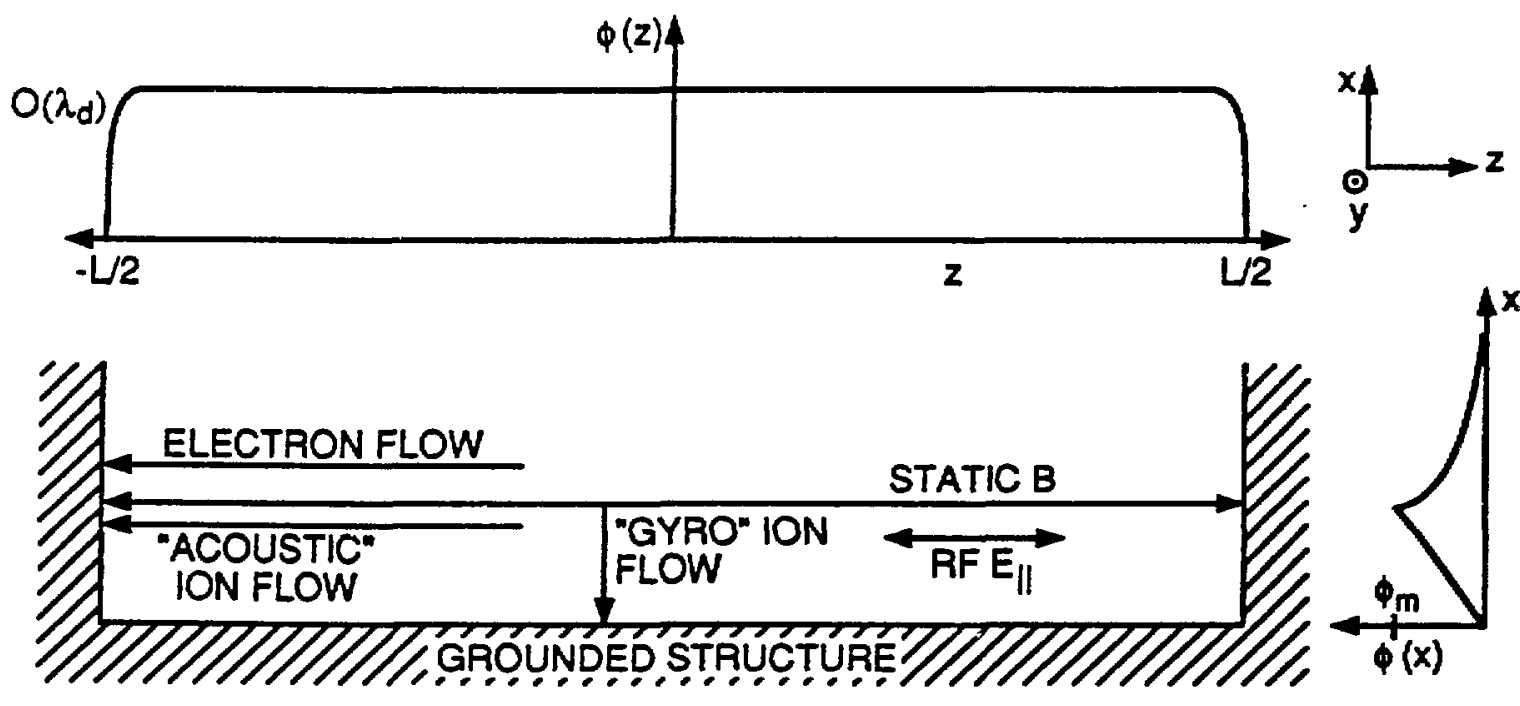

FIG. I 


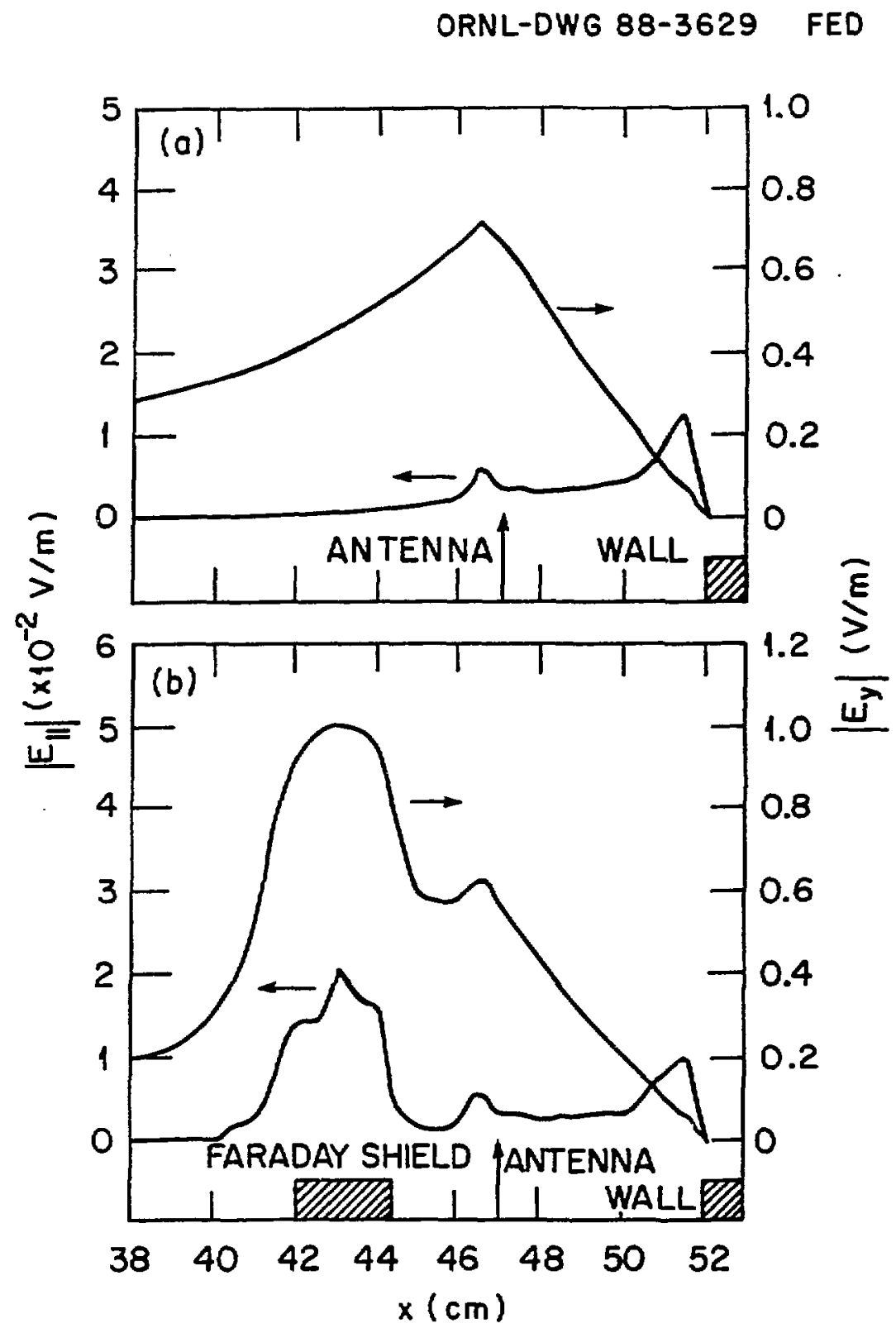

FIG. 2 


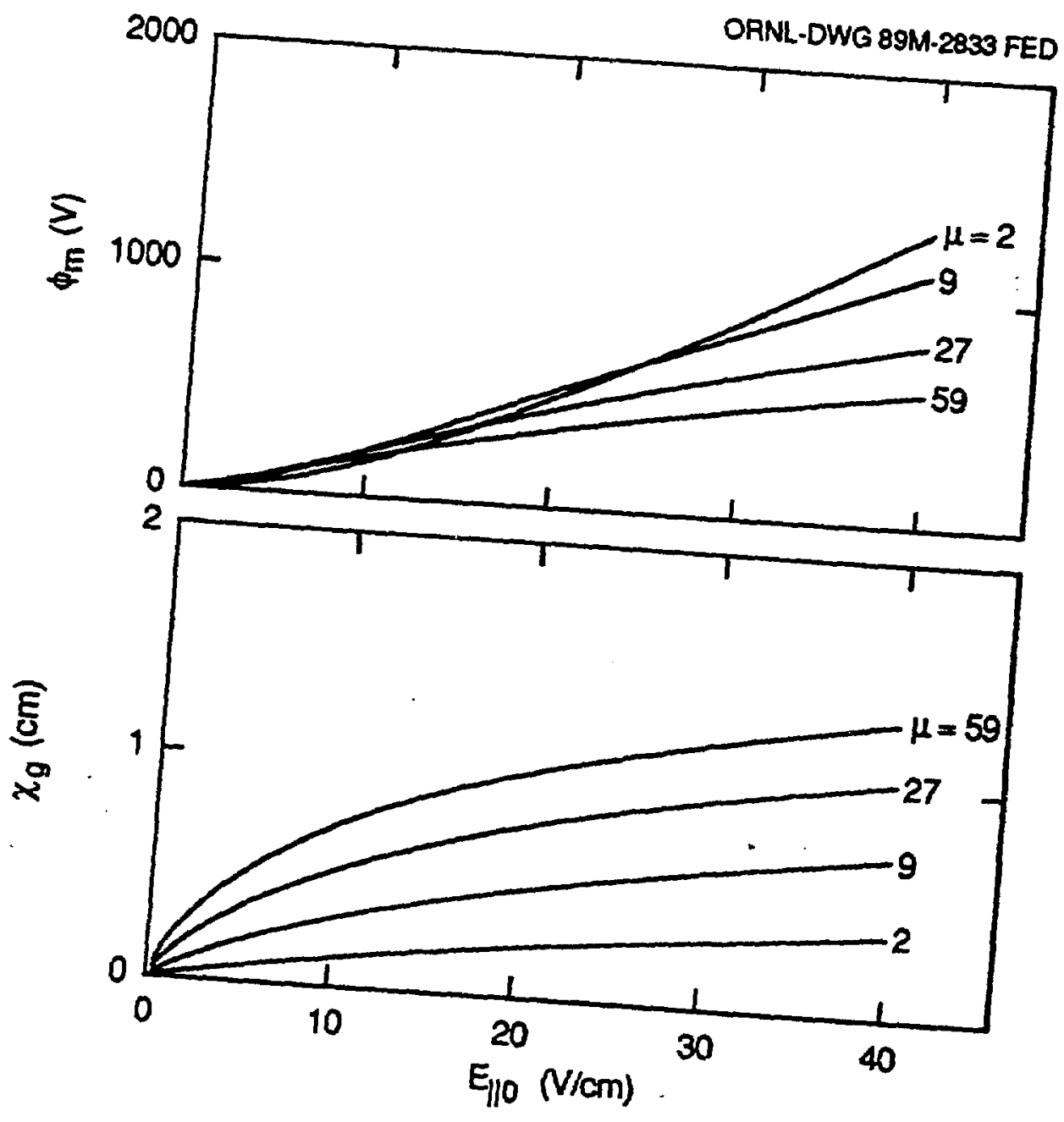

FIG. 3 


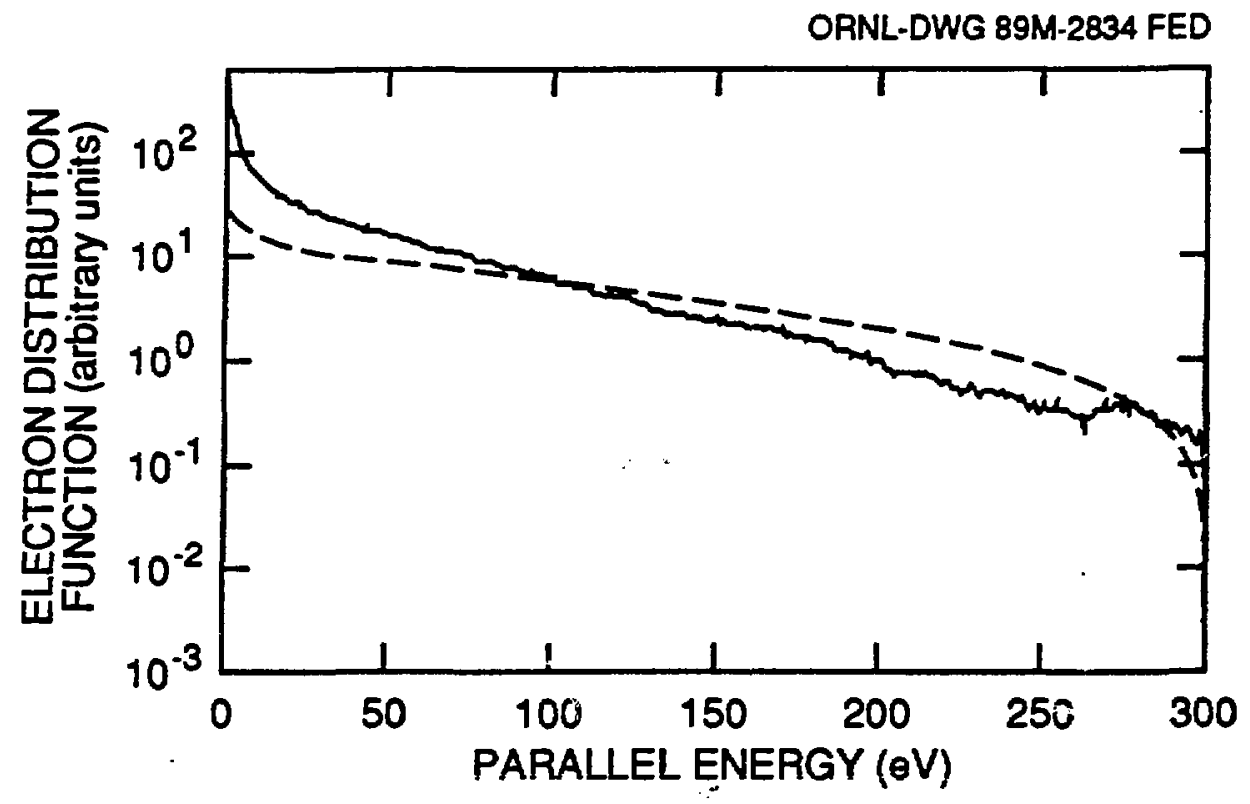

FIG. 4 